\title{
Experimental and numerical analysis of atmospheric propagation of high energy laser
}

\author{
Carsten Pargmann*a, Thomas Hall ${ }^{\mathrm{b}}$, Frank Duschek ${ }^{\mathrm{a}}$, Thomas Fischbach ${ }^{\mathrm{a}}$, Karin M. Grünewald ${ }^{\mathrm{a}}$, \\ Kirsten Klaffki ${ }^{a}$, Jim Thieser ${ }^{a}$, Jürgen Handke ${ }^{a}$ \\ ${ }^{a}$ German Aerospace Center (DLR), Institute of Technical Physics, Langer Grund, \\ D-74239 Hardthausen, Germany; \\ ${ }^{\mathrm{b}}$ German Aerospace Center (DLR), Institute of Technical Physics, Pfaffenwaldring 38-40, \\ D-70569 Stuttgart, Germany
}

\begin{abstract}
The transmission of high power laser radiation through the air is influenced by atmospheric turbulence. As a result the beam experiences variations regarding its position and its distribution, which increase with increasing propagation length. In order to analyze the atmospheric influence on the laser beam propagation a disk laser with a maximum output power of $6 \mathrm{~kW}$ and a wavelength of $1.03 \mu \mathrm{m}$ is operated on the $130 \mathrm{~m}$ long free transmission laser test range at Lampoldshausen. The test range is equipped with a variety of sensors, which continuously monitor the current status of the weather conditions. Power sensors and camera systems at the beginning and the end of the test range measure the laser beam parameters before and after propagation.

First measurements of atmospheric power transmission, diameter change of the laser beam and deviation of its center of gravity are performed on a sunny and on a rainy day and are compared with turbulence strength, visibility and rainfall. The results show good correlation between the optical parameters and the weather conditions.

Following measurements will be performed at different weather conditions and seasons. Experimental results will be compared to a numerical analysis.
\end{abstract}

Keywords: high energy laser, atmospheric propagation, disk laser, laser beam characterization

\section{INTRODUCTION}

The directed wireless transmission of high power densities through the atmosphere is of interest for numerous applications. Security relevant examples include the powering of aircrafts and satellites as well as selective counter measures against hostile projectiles ${ }^{1}$. The advent of high energy laser systems with emission wavelengths in the near infrared range reduced the effect of atmospheric absorption. Nevertheless, the laser beam is still exposed to beam wandering, beam deterioration and scattering processes ${ }^{2,3}$. Due to the accumulation of these effects with propagation distance the optical quality of the laser beam is severely degraded. As a result the power density and the efficiency of the atmospheric laser transmission are largely reduced.

In order to analyze the atmospheric influence on the laser beam propagation the German Aerospace Center (DLR) operates a free transmission laser test range at Lampoldshausen. The beam of a disk laser propagates in the height of $1 \mathrm{~m}$ above asphalt ground. The path length is $130 \mathrm{~m}$ between transmitting station (TS) and receiving station (RS). A variety of sensors continuously monitor the atmosphere in order to get a detailed picture of the current status of the weather conditions. The test facility is operational at all weather conditions. The TS and RS contain sensors to measure the transmitted power of the laser beam and cameras to measure beam wandering, power-in-the-bucket, and beam distortion.

The intention is to demonstrate the feasibility of the optical setup by showing a correlation between weather condition and optical parameters. 

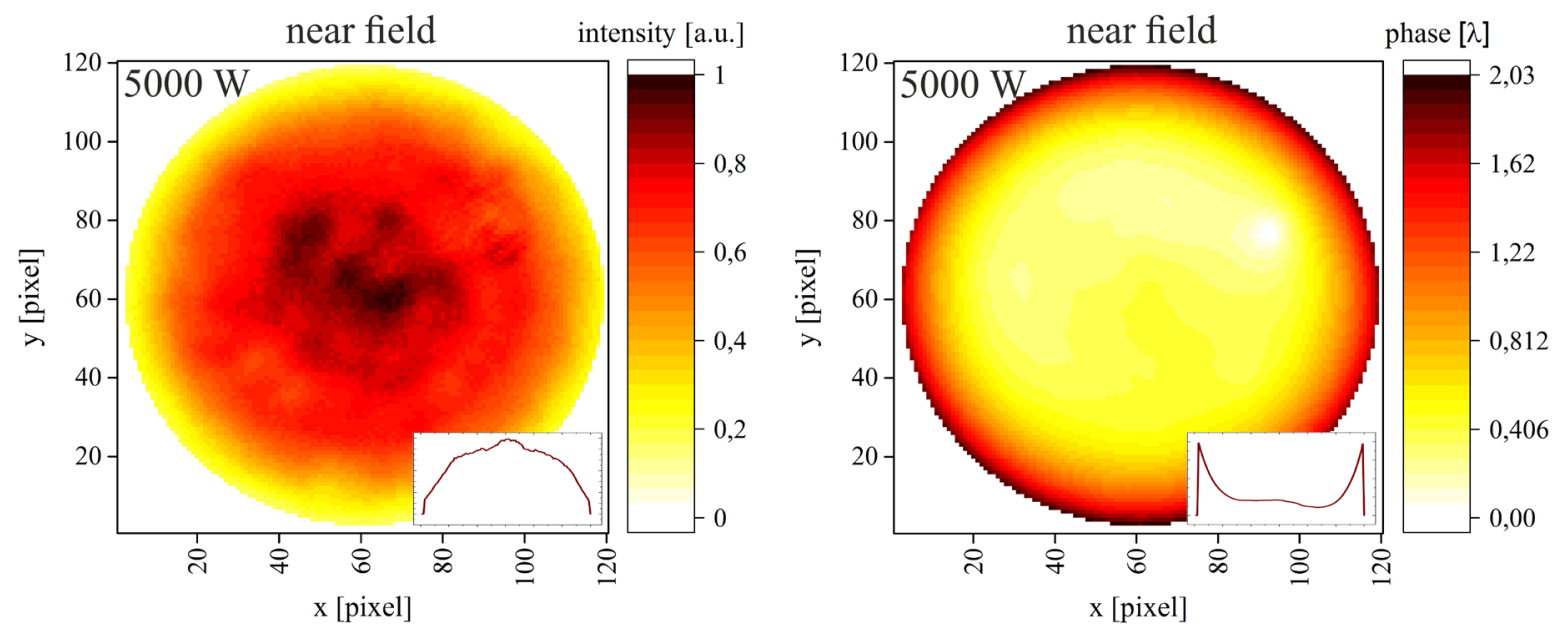

Figure 1. Intensity (left) and phase (right) distribution of the near field of the disk laser at $5 \mathrm{~kW}$ output power after propagation through a $40 \mathrm{~m}$ long optical fiber and a collimation lens of $100 \mathrm{~mm}$ focal length.

\section{LASER SYSTEM}

Powered by industrial demands solid-state high power lasers are getting more and more sophisticated. Hence, nowadays commercially available laser systems reach power levels of $10 \mathrm{~kW}$ and above with noteworthy beam qualities ${ }^{4,5}$. These systems deliver high power stability and availability and operate in the $1 \mu \mathrm{m}$ wavelength region, yielding a high transmission through the atmosphere. Due to these specifications solid-state high power laser systems are also interesting for non-industrial applications.

The laser source used here is the TruDisk 6001 (4C), a disk laser of the Trumpf company. The emitted wavelength is $1.03 \mu \mathrm{m}$, the output power (continuous wave) is adjustable between $180 \mathrm{~W}$ and $6 \mathrm{~kW}$ and the beam quality is specified to be $4 \mathrm{~mm} \cdot \mathrm{mrad}$. The laser device is located in a laboratory outside of the free transmission laser test range. Hence the laser radiation is coupled to a $40 \mathrm{~m}$ long optical fiber with a core diameter of $100 \mu \mathrm{m}$ and transferred to the transmitting station.

In order to ensure a most realistic comparison between experiment and numerical simulations the intensity and phase distribution of the near field of the disk laser is measured at a plane positioned right after a collimation lens with a focal length of $100 \mathrm{~mm}$ at the end of the optical fiber in the transmitting station. The optical measurement setup consists of a relay telescope including two lenses with focal lengths of $1500 \mathrm{~mm}$ and $300 \mathrm{~mm}$, respectively. The magnification factor is about 0.2 . The wavefront sensor SID4 of the company Phasics provides simultaneous information about the intensity and the phase distribution. Both distributions are cut at the diameter embedding $86 \%$ of the total power. In Figure 1 the measurements for a power of $5 \mathrm{~kW}$ are shown. As a result the diameter of the laser beam is about $18.3 \mathrm{~mm}$ at the output of the collimation lens. The obtained distributions can be used as initial distributions for following numerical analyses.

The measurements of the near field display an increase of the diameter of the intensity distribution with increasing power of the laser beam. Therefore the beam propagation factor $\mathrm{M}^{2}$ is also measured at different power levels using a lens with a focal length of $307 \mathrm{~mm}$ and the beam diagnostics camera Beamage-3.0 of the company Gentec. In order to measure the caustic near to the focal point of the lens the camera is positioned on a linear translation stage. In Figure 2 the intensity distribution of the far field and the change of the diameter of the second moments with the change of the camera position are shown. The beam propagation factor $\mathrm{M}^{2}$ is obtained to be 14.2 at a power level of $5 \mathrm{~kW}$. This value is slightly exceeding the $4 \mathrm{~mm} \cdot \mathrm{mrad}$. But lowering the output power also leads to lower beam propagation factors, lying well below the specified value. 

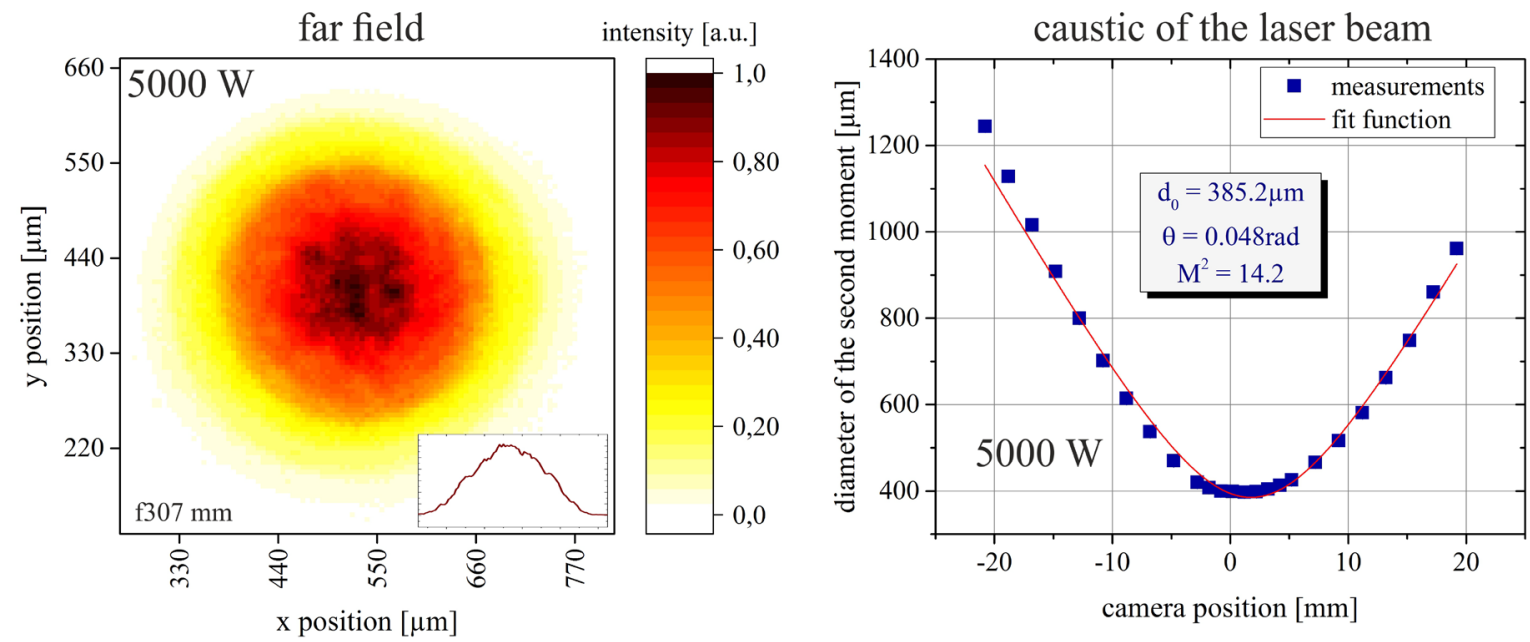

Figure 2. Far field intensity distribution in the focus of a lens with a focal length of $307 \mathrm{~mm}$ (left) and the change of the second moment diameter with the change of the camera position (right).

\section{FREE TRANSMISSION LASER TEST RANGE}

In Figure 3 a view onto the free transmission laser test range is shown. The propagation path of the laser beam is arranged to be $1 \mathrm{~m}$ above an asphalt route of $130 \mathrm{~m}$ length. The distance for atmospheric propagation can be increased up to the kilometer range by optical folding of the laser beam.

An air conditioned transmitting station (TS) and a receiving station (RS) are the structural boundaries at the start and the end of the propagation path. Each station is equipped with $100 \mathrm{~kW}$ of electrical power and an additional uninterruptible power supply (UPS). Earth walls on both sides of the propagation path protect the region outside the test range against direct or indirect laser radiation. The energy supply along the propagation path is completed by three energy columns delivering $30 \mathrm{~kW}$ of electrical power, each.

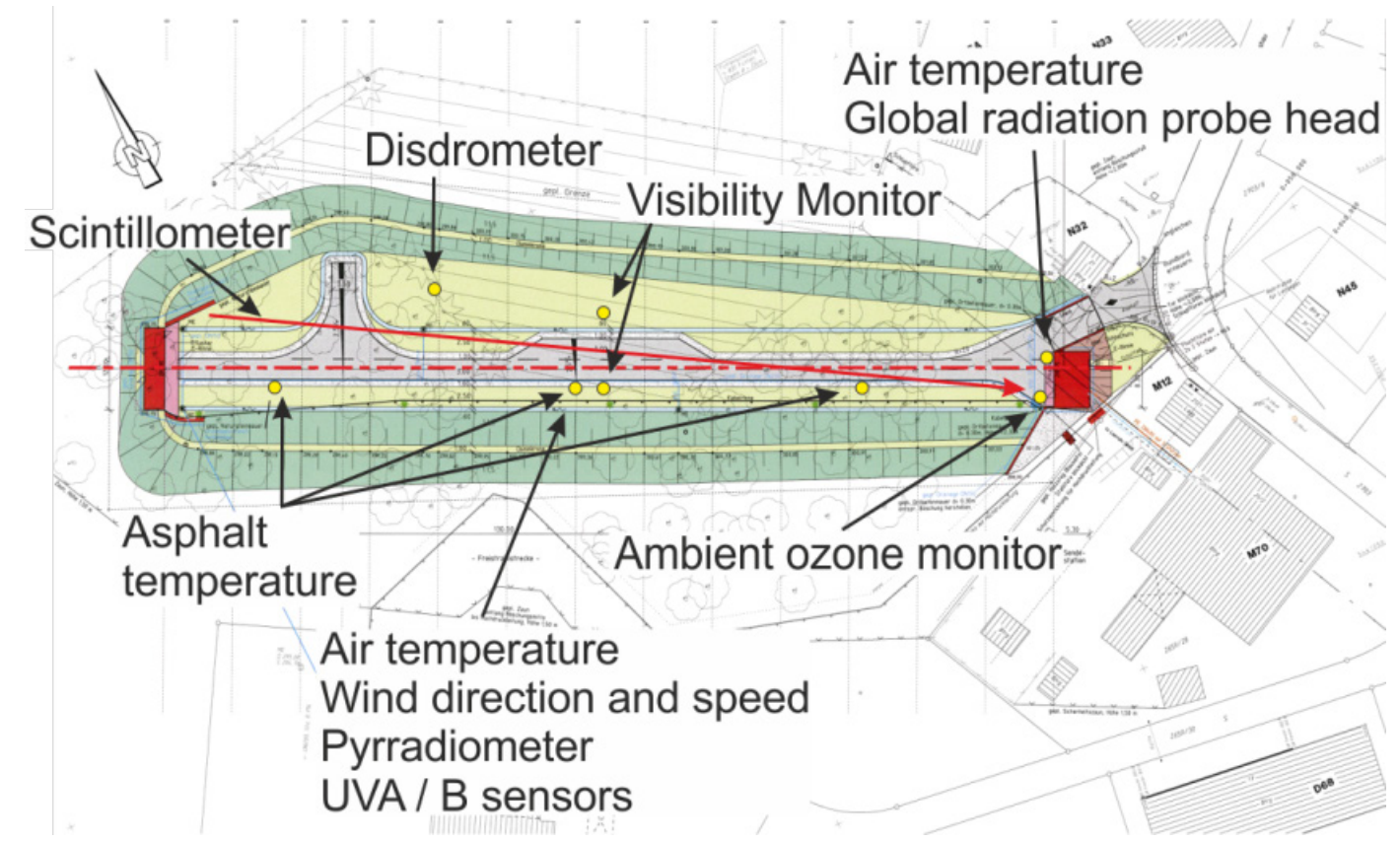

Figure 3. Top view onto the free transmission laser test range and some of its sensors. 
A large set of detectors is located around the propagation path. These detectors monitor atmospheric conditions such as temperature, pressure, relative humidity, precipitation, wind, and visibility. Additional detectors measure radiation data from the ultraviolet up to the far-infrared region and a scintillometer measures the turbulence strength and the inner scale of the turbulent cells. An aerosol spectrometer is also installed and gives information on the distribution of aerosol particles for a better analysis of laser radiation scattered at them.

Propagation measurements have to be performed at different atmospheric conditions. These include diverse types of rain, fog, haze, snow, dust and other anomalous conditions. The detectors monitoring these atmospheric conditions operate night and day, independent of other activities.

\section{EXPERIMENTAL SETUP}

The disk laser is operated in a laboratory next to the free transmission laser test range and the laser beam is coupled to an optical fiber. The end of the optical fiber is positioned on an optical table inside of the TS of the test range. In Figure 4 the optical setup inside of the TS is shown. Right after the end of the optical fiber the laser beam is collimated by a lens with a focal length of $100 \mathrm{~mm}$ and the beam has a diameter of $18.3 \mathrm{~mm}$ at $5 \mathrm{~kW}$ (Figure 1). Three plane mirrors guide the beam into a telescope consisting of two concave off-axis parabolic mirrors with focal lengths of $227 \mathrm{~mm}$ and $2272 \mathrm{~mm}$, respectively, expanding the beam diameter up to approximately $183 \mathrm{~mm}$. The distance of the telescope mirrors is adjusted to yield the focus point in a distance of $135 \mathrm{~m}$, inside of the RS. The off-axis parabolic mirrors reduce the effect of astigmatism. Furthermore the telescope directs the beam onto a plane mirror with a diameter of $300 \mathrm{~mm}$. This mirror is mounted on two direct-drive rotary stages. The laser beam is guided computer controlled onto the free transmission laser test range towards the RS. Just before the main beam leaves the TS a part of the laser energy is reflected by an optical wedge towards a measurement setup. This setup consists of an off-axis parabolic mirror, a Beamage-3.0 camera of the company Gentec positioned in the focus of the beam, a thermopile sensor, and an integrating sphere with a photo diode. The camera records intensity distribution and center of gravity of the far field of the laser beam, the thermopile sensor measures the absolute power of the beam, and the photo diode records fast energy changes up to the kHz-range. The photo diode is calibrated by the integrated signal of the thermopile.

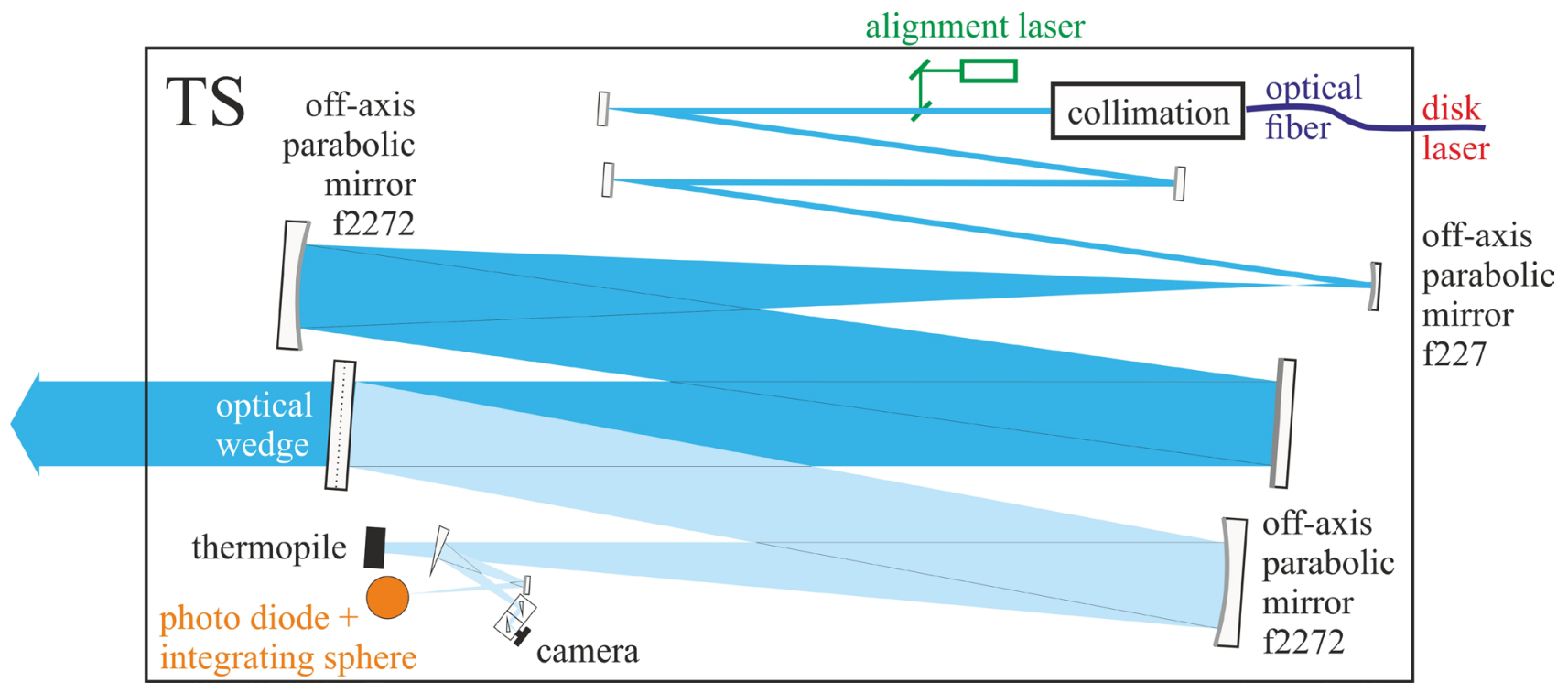

Figure 4. Optical setup inside of the transmitting station TS. 

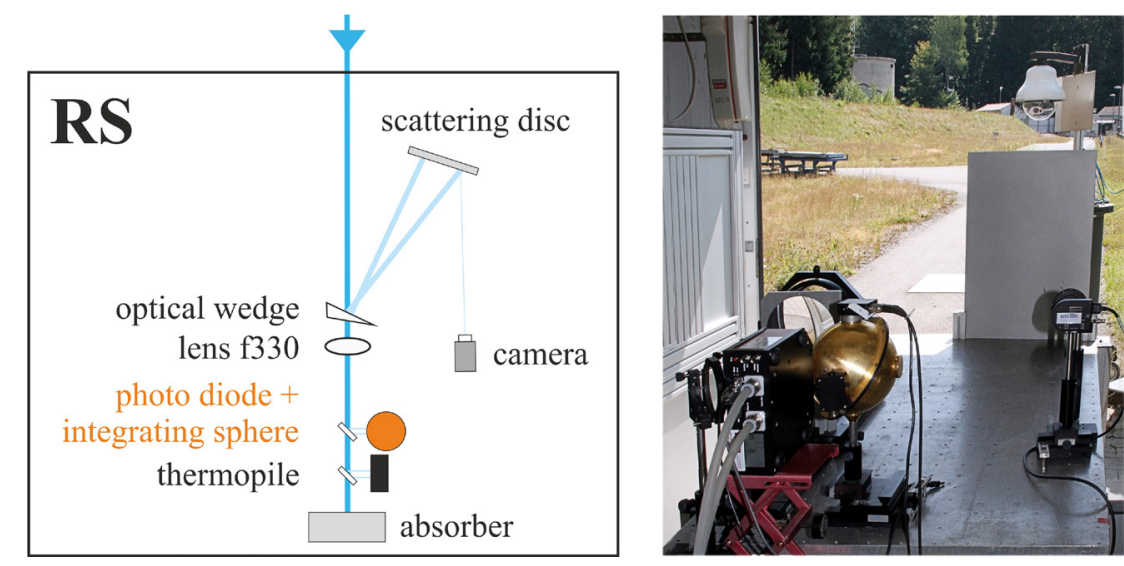

Figure 5. Optical setup inside of the receiving station RS (left) and photo of the setup (right).

The optical setup inside of the RS is shown in Figure 5 together with a photo of the setup. The main beam is directed onto an absorber. But before, parts of the energy are reflected onto a thermopile, an integrating sphere with a photo diode, and onto a scattering disk. The free aperture of the optics is $140 \mathrm{~mm}$. The intensity distribution onto the scattering disk is recorded by a Beamage-3.0 camera measuring the intensity distribution and the center of gravity of the laser beam. As in the TS the thermopile sensor and the photo diode record the absolute power and fast energy changes of the laser beam, respectively.

A combination of all measurements in TS and RS yields conclusions about the influence of the atmosphere onto the propagation of the laser beam. The parameters to be compared include power transmission through the atmosphere, change of the diameter of the laser beam (based on $86 \%$ of power content) and standard deviation of the position of the center of gravity of the laser beam during the measurements. All measurements are performed at $5 \mathrm{~kW}$ and last for $10 \mathrm{~s}$. The frame rate of the cameras is about $10 \mathrm{~Hz}$ and the exposure time is below $1 \mathrm{~ms}$. This low exposure time allows to differentiate between two different effects of the atmosphere onto the laser beam: a change of the intensity distribution and a change in the position of the center of gravity.

\section{RESULTS}

For a first test of the optical setup measurements are performed on two days of different weather conditions, a rainy and a sunny one. Figure 6 shows the main results obtained on the rainy day. The graphs are labeled as rainfall, turbulence strength, visibility, power difference TS-RS and effective diameter RS-TS.

The rainfall is measured by a disdrometer and provides a precipitation intensity. The turbulence strength is measured along the asphalt route of the test range by a scintillometer, and the visibility is obtained by measuring the attenuation of a super wide band diode along a path of $20 \mathrm{~m}$. The positions of the weather sensors are shown in Figure 3.

The power difference TS-RS denotes the subtraction of the laser power measured by the thermopile in the receiving station RS from the power measured in the transmitting station TS. In order to get the power levels at the positions where the laser beam just leaves the TS and where the beam just enters the RS the respective optical correction factors are applied. The effective diameter RS-TS is obtained by measuring at first the diameter containing $86 \%$ of the total power around the respective center of gravity of approximately 100 intensity distributions in TS and RS. The calculated average diameter in the TS is multiplied by the ratio of the corresponding focal lengths and is subtracted from the average diameter calculated for the receiving station. Hence the displayed parameter "effective diameter RS-TS" is a measure for the change of the average diameter of the laser beam induced by the atmosphere. The measurements of the rainy day show two correlations: at first between the effective diameter change in the RS and the rainfall, and at second between the visibility and the power transmission loss. The second correlation is obtained despite of the large errors bars of the power difference values of approximately $200 \mathrm{~W}$. 

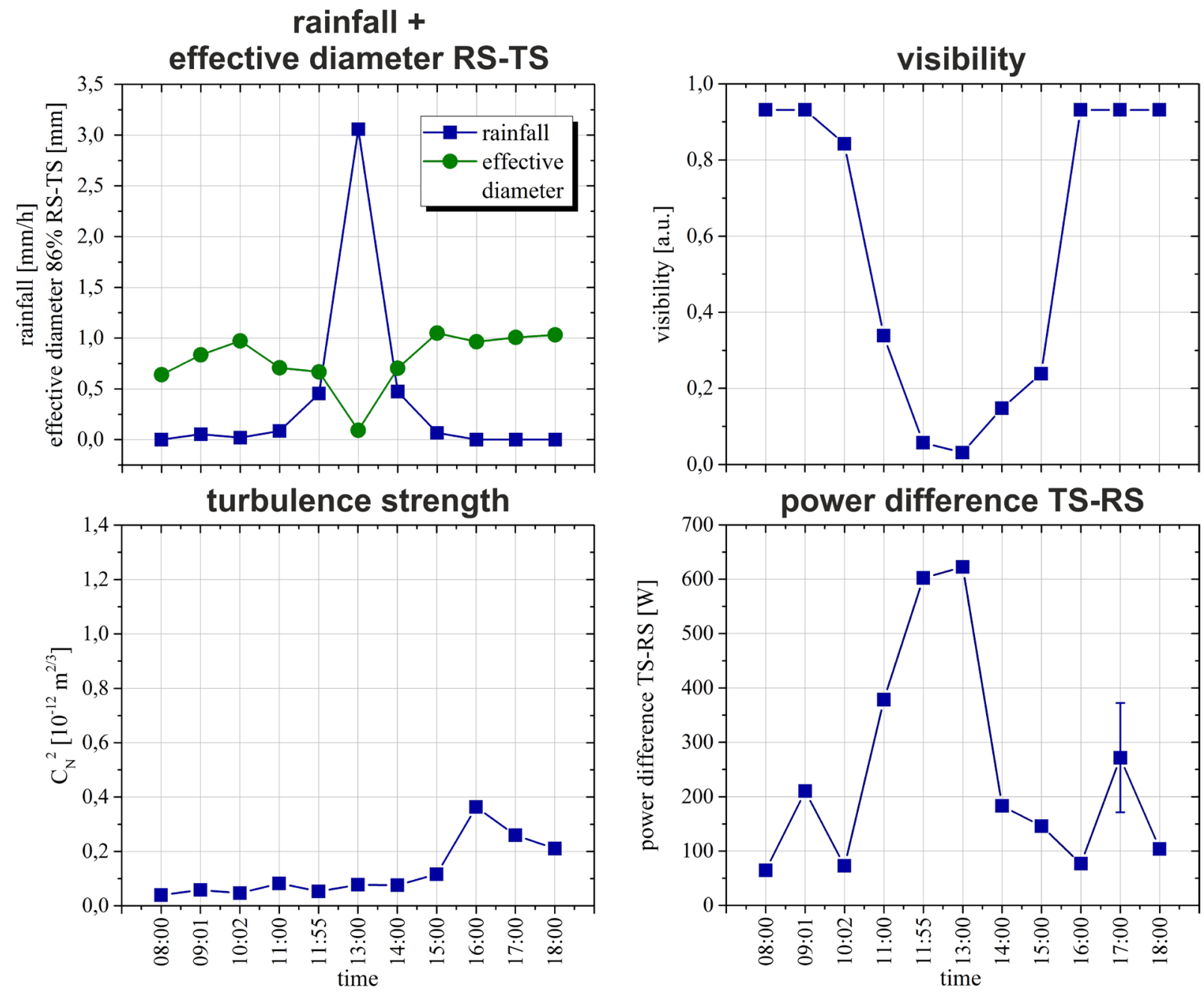

Figure 6. Atmospheric and optical data measured on a rainy day.

The results for the sunny day are shown in Figure 7. The graphs show the turbulence strength, the temperatures of the asphalt and of the air $1 \mathrm{~m}$ above ground, the effective diameter RS-TS and the deviation of the center of gravity in the RS. Turbulence strength and effective diameter RS-TS are obtained as described above. The temperatures are measured at the middle of the transmission range. The deviation of the center of gravity is obtained by measuring the respective position of the center of gravity of 100 intensity distributions inside of both stations. Afterwards the calculated standard deviation of the center of gravity inside of the TS is subtracted from the one obtained in the RS. As a result the deviation of the center of gravity displays mainly the influence of the atmosphere. The deviations of the horizontal and of the vertical direction, as well as the average of both directions are shown. Again two correlations between weather and laser beam parameters can be seen: between the turbulence strength and the change of the effective diameter in the RS and between the turbulence strength and the deviation of the center of gravity. However the variation of the center of gravity can be reduced by the application of active optical systems with tip/tilt correction.

\section{CONCLUSION AND OUTLOOK}

In order to measure the influence of the atmosphere on the propagation of a high power laser beam a free transmission laser test range is built up and equipped with sensors for a comprehensive recording of the weather conditions. Data like optical turbulence, visibility and rainfall are monitored simultaneously to the measurement of laser beam parameters, like power transmission loss, intensity distribution, and center of gravity of the beam. First results obtained on days with different weather conditions not only display the technical feasibility of the measurements, but also the direct correlation between weather conditions and laser beam parameters. 

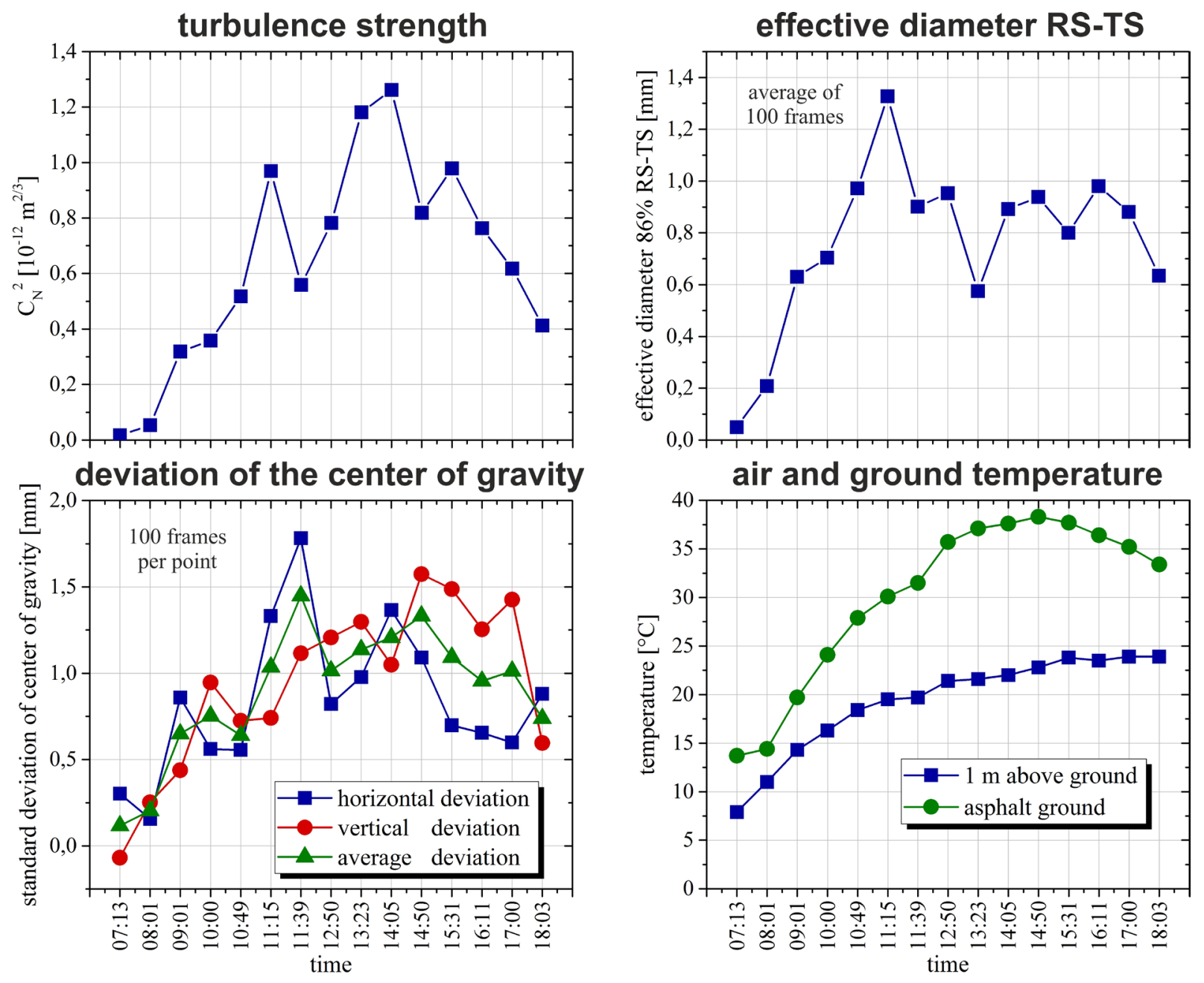

Figure 7. Atmospheric and optical data measured on a sunny day.

Ongoing measurements will be performed under a variety of conditions, in order to get a better statistics and to be able to better specify these correlations. For a better classification and understanding of the experimental results and in order to be able to extrapolate the relations to different propagation scenarios the atmospheric laser beam propagation will be analyzed numerically. Parts of these accompanying analyses will be performed by the software TALAP (Turbulent Atmosphere and Laser Beam Propagation), developed at the Institute of Technical Physics of DLR.

\section{REFERENCES}

[1] Fiorino, S., Bartell, R. J., Krizo, M. J., and Cusumano, S. J., "Propagation variability assessments of ship defense HEL and HPM performance in worldwide maritime boundary layer environments at wavelengths of $1.0642 \mu \mathrm{m}$, $2.141 \mu \mathrm{m}, 3.16 \mathrm{~mm}$ and $12.2 \mathrm{~cm}$," Poc. SPIE 6951, G9510-G9510 (2008).

[2] Weichel, H., “Atmospheric propagation of laser-beams," Proc. SPIE 547, 1-15 (1985).

[3] Sprangle, P., Ting, A., Penano, J., Fischer, R., and Hafizi, B., "Incoherent Combining and Atmospheric Propagation of High-Power Fiber Lasers for Directed-Energy Applications," IEEE J. Quant. Electron. 45(1-2), 138-148 (2009).

[4] Giesen, A., Hügel, H., Voss, A., Wittig, K., Brauch, U., and Opower, H., "Scalable concept for diode-pumped highpower solid-state lasers,“ Appl. Phys. B 58(5), 365-372 (1994).

[5] Limpert, J., Röser, F., Klingebiel, S., Schreiber, T., Wirth, C., Peschel, T., Eberhardt, R., and Tünnermann, A., „The rising power of fiber lasers and amplifiers,“ IEEE J. Sel. Top. Quantum Electron. 13(3), 537-545 (2007). 